\title{
Laparoscopic Pancreatectomy for Pancreatic Cancer
}

\section{Yoshiharu Nakamura*, Akira Matsushita, Hiroki Sumiyoshi, Kazuya Yamahatsu, Takayuki Aimoto and Eiji Uchida}

Department of Gastrointestinal and Hepato-Biliary-Pancreatic Surgery, Nippon Medical School, Tokyo, Japan

\begin{abstract}
Introduction: The recent advances of surgical techniques and technology allow minimally invasive surgery to be applied in patients with benign and malignant diseases of the pancreas. About malignancy, we still have concerns regarding the oncologic adequacy of laparoscopic pancreatectomy, with fewer studies reporting oncologic outcomes. We describe the surgical technique to improve the curability of laparoscopic pancreatectomy in the treatment of Pancreatic Adenocarcinoma (PDAC) and focuse on the oncologic outcomes and long-term outcomes of laparoscopic surgery for PDAC patients.

Methods: From January 2004, patients who had been diagnosed with the tumor in the pancreas without suspicion for vascular involvement were eligible for laparoscopic pancreatectomy at Nippon Medical School. In LapPD for PDAC patients, we apply laparoscopic left mesenteric approach, which enables both accurate laparoscopic lymph node retrieval and complete pancreatic nerve plexuses dissection to achieve R0 resection. In Lap-DP for PDAC patients, we perform retroperitoneal tissue dissection, which often includes adrenalectomy

Results: We have experienced laparoscopic pancreatectomies in 148 patients including 25 PDAC patients. In the 25 patients with PDAC, the mean number of lymph nodes dissected was $22.4 \pm 12.6(6-57)$. Metastasis to the lymph nodes was observed in 8 of the 25 patients (32\%). R0 resection was performed in 22 patients (88\%). The median follow-up period for the PDAC patients undergoing laparoscopic pancreatectomy was 16 months (1-71 months). Six of the 25 patients died, at 2.5 months (stage IV), 15 months (stage IA), 29 months (stage IIB), 33 months (stage IIB), 24 months (stage IIA), and 18 months (stage IIB). Surviving 19 patients had no recurrence.
\end{abstract}

Conclusion: Laparoscopic pancreatectomy for pancreatic cancer seems to achieve similar oncologic and longterm outcomes to open approach.

Keywords: Laparoscopic pancreatectomy; Pancreatic cancer; Laparoscopic left mesenteric approach; Prevention of tumor cell dissemination

\section{Introduction}

The recent advances of surgical techniques and technology allow minimally invasive surgery to be applied in patients with benign and malignant diseases of the pancreas. Whereas laparoscopic distal pancreatectomy (Lap-DP) is commonly performed minimally invasive pancreatic resection due to no requirement of anastomosis, laparoscopic pancreaticoduodenectomy (Lap-PD) less studied and limited to experienced surgeons and institutions according to the complexity of dissection and necessity of reconstruction with three major anastomoses.

Concerning malignancy, we still have concerns regarding the oncologic adequacy of laparoscopic pancreatectomy, with fewer studies reporting oncologic outcomes such as margin status, lymph node retrieval and survival. Surgeons should take it into consideration that Pancreatic Adenocarcinoma (PDAC) is highly biologically malignant compared with other gastrointestinal cancers. Therefore, when performing laparoscopic surgery for PDAC, surgeons need to improve surgical procedures that achieve equivalent oncologic outcomes to open approach. Laparoscopy could magnify intra-abdominal tissues, organs and vessels 10 to 15 times comparing with open approach. Laparoscopic surgery can improve visualization of intraabdominal organs especially retroperitoneal organs, such as the pancreas, its neighboring vessels, the duodenum, the adrenal grand and the kidney for all surgeons involved in the operation. In this respect, laparoscopic pancreatic surgery is obviously superior to open approach, in which the ribs and anterior organs to the pancreas obstruct the operative field. Moreover, improved visualization also enables precise dissection using forceps. It is important to create the good operative field when dissecting the lymph nodes and pancreatic nerve plexuses where cancer cells easily infiltrate and perform $\mathrm{R} 0$ resection resulting in an increase of the long-term survivors of PDAC. On the other hand, the disadvantage of laparoscopic surgery compared with open approach is that there are limited range of motion and limited sense of touch, because laparoscopic surgery is performed using several forceps inserted through trocars.

Here, we discuss the surgical technique for laparoscopic pancreatic surgery surgery for malignancy. We also focused on the oncologic outcomes and long-term outcomes of laparoscopic surgery for PDAC patients.

\section{Patients and Methods}

From January 2004, patients who had been diagnosed with the tumor in the pancreas without suspicion for vascular involvement were eligible for laparoscopic pancreatectomy at Nippon Medical School. Informed consents were obtained from all of patients. Detailed descriptions of the techniques we use for Lap-DP and Lap-PD were already reported [1-3].

*Corresponding author: Yoshiharu Nakamura, Department of Gastrointestina and Hepato-Biliary-Pancreatic Surgery, Nippon Medical School, 1-1-5 Sendagi, Bunkyo-ku, Tokyo 113-8603, Japan, Tel: +81 33822 2131; Fax: +81 356850989 E-mail: keishun@nms.ac.jp

Received March 11, 2013; Accepted June 10, 2013; Published June 17, 2013

Citation: Nakamura Y, Matsushita A, Sumiyoshi H, Yamahatsu K, Aimoto T, et al. (2013) Laparoscopic Pancreatectomy for Pancreatic Cancer. J Carcinogene Mutagene S9: 004. doi:10.4172/2157-2518.S9-004

Copyright: (c) 2013 Nakamura Y, et al. This is an open-access article distributed under the terms of the Creative Commons Attribution License, which permits unrestricted use, distribution, and reproduction in any medium, provided the original author and source are credited. 
Citation: Nakamura Y, Matsushita A, Sumiyoshi H, Yamahatsu K, Aimoto T, et al. (2013) Laparoscopic Pancreatectomy for Pancreatic Cancer. J Carcinogene Mutagene S9: 004. doi:10.4172/2157-2518.S9-004

In Lap-PD for PDAC patients, we apply laparoscopic left mesenteric approach, which enables both accurate laparoscopic lymph node retrieval and complete pancreatic nerve plexuses dissection to achieve R0 resection owing to the magnified operative field (Figures 1a,1b and 2). Moreover, we use Endoscopic Linear Stapler (ELS) when cutting the pancreas during Lap-PD in order to avoid the leakage of pancreatic juice, which may contain tumor cells from the neoplastic lesion [3] (Figure 3).

In order to execute an accurate R0 resection in Lap-DP for PDAC patients, we perform retroperitoneal tissue dissection, which often includes adrenalectomy (Figure 4). We could create the good operative field, compared with open distal pancreatectomy in which the left costal arch overhangs the operative field.

\section{Results}

We have performed laparoscopic pancreatectomy in 148 patients from January 2004 to December 2012, laparoscopic distal pancreatectomy (Lap-DP) in 98 patients, laparoscopic pancreaticoduodenectomy (Lap-PD) in 40 patients, laparoscopic central pancreatectomy in 6 patients, and laparoscopic enucleation in 4 patients.

From January 2004 to December 2012, 35 patients who were diagnosed with malignant pancreatic diseases as primary lesions underwent laparoscopic pancreatectomy in our department. This subgroup of patients were treated with either Lap-PD ( $\mathrm{n}=11)$ or LapDP $(n=24)$. PDAC was diagnosed in 25 patients ( 10 males, 15 females) with a mean age of 67.3 years (29-88 years). TNM classification of the disease stage showed 3 patients at stage 0,5 patients at stage IA (1 patient after neo-adjuvant chemotherapy), 2 patients at stage IB, 5 patients at stage IIA, 9 patients at stage IIB, and 1 patient at stage IV (Table 1). Of the 10 patients were not PDAC, there were 5 patients with metastatic tumor of the pancreas, 4 patients with neuro-endocrine carcinoma, and 1 patient with another type of malignancy.

In the 25 patients with PDAC treated with laparoscopic pancreatectomy, the mean number of lymph nodes dissected was 22.4 \pm 12.6 (6-57). Metastasis to the lymph nodes was observed in 8 of the
25 patients (32\%). R0 resection was performed in 22 patients (88\%). The median follow-up period for the PDAC patients undergoing laparoscopic pancreatectomy was 16 months (1-71 months). Six of the 25 patients died, at 2.5 months (stage IV), 15 months (stage IA), 29 months (stage IIB), 33 months (stage IIB), 24 months (stage IIA), and 18 months (stage IIB) (Table 1). All of PDAC patient received adjuvant chemotherapy and surviving 19 patients had no recurrence.

\section{Discussion}

In terms of the oncologic outcomes with Lap-DP, there are a number of papers comparing this procedure with open approach [913]. Jayaramen et al. reported that in terms of the number of lymph nodes retrieved, there were no differences between Lap-DP and openDP when patients were matched for malignancy, tumor size, and age 10. They also reported a satisfactory rate of $\mathrm{R} 0$ resections regardless of approach. Mehta et al. [13] reported that there was no difference in the mean number of lymph nodes harvested in their series with equal proportions of patients with pancreatic cancer in both the Lap-DP and open-DP groups. However, the long-term prognoses were difficult to evaluate because many of the series included a mix of benign and malignant cases. Kooby et al. [11] published a multicenter analysis evaluating the oncologic safety of Lap-DP, compared to open-DP for pancreatic adenocarcinoma. They reported no significant differences in positive margin rates, number of nodes examined, number of patients with at least 1 positive node, or overall survival in their matched analysis. In a review of laparoscopic pancreatectomy for malignancy, Fisher and Kooby reported that, based on the existing information, it appears that Lap-DP can achieve similar short-term oncologic outcomes, with comparable rates of margin-positive resections and numbers of lymph nodes retrieved, and potentially equivalent long-term survival [14]

On the other hand, in terms of the oncologic outcomes with Lap$\mathrm{PD}$, in five published reports that included at least five patients with PDAC, the mean lymph nodes procured ranged between 12 and 20.8 nodes [4-8]. Kendrick et al. [7] reported on 65 patients including 32 with PDAC, where the mean number of lymph nodes harvested was 15 , ranging between 6 and 31 nodes and positive margins in $11 \%$ of patients. The remaining four papers all reported positive margins in

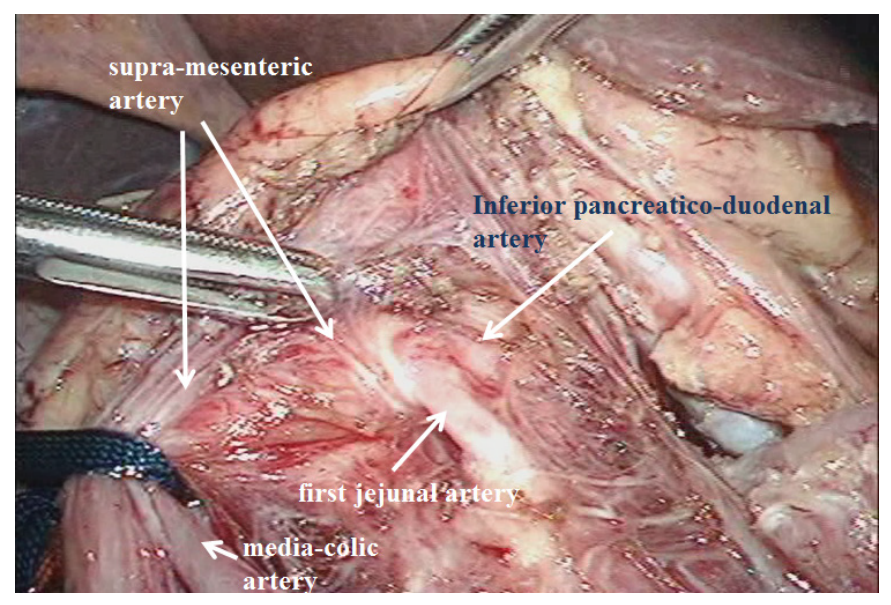

a

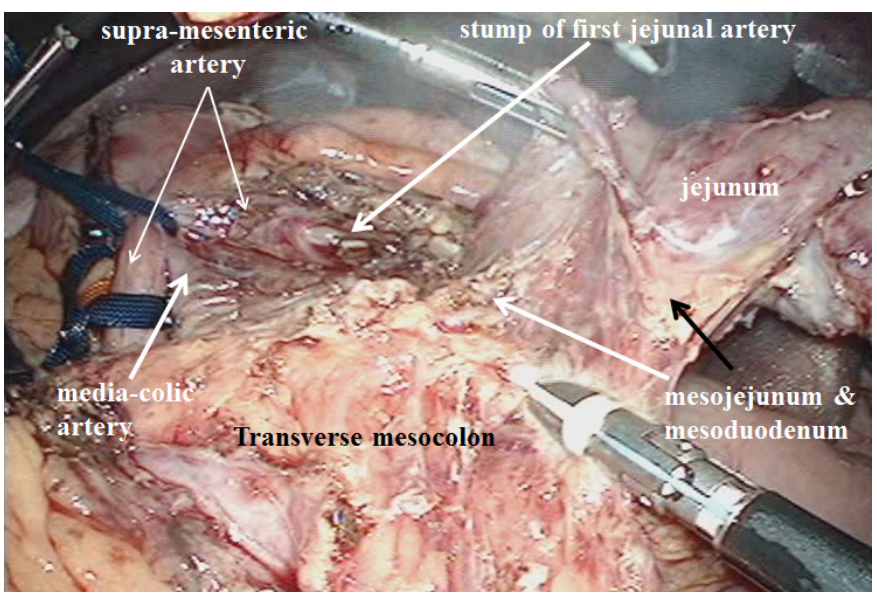

b

Figure 1: Laparoscopic left mesenteric approach. Following the Treitz ligament and transverse mesocolon are cut, the jejunum is passed to the supracolic compartment on the left side of the Supra-Mesenteric Artery (SMA). The jejunum is then retracted to the left. While the assisting surgeon gently retracts the SMA to the right, the nerve plexus and blood vessels between the head of the pancreas and SMA are clearly exposed (Figure 1a). Following the jejunum is transected, the mesojejunum and mesoduodenum are resected on the border between transversemesocolon and those mesenteries toward the root of the media-colic artery by vessel sealing system or tissue sealing device (Figure 1b). 
Citation: Nakamura Y, Matsushita A, Sumiyoshi H, Yamahatsu K, Aimoto T, et al. (2013) Laparoscopic Pancreatectomy for Pancreatic Cancer. J Carcinogene Mutagene S9: 004. doi:10.4172/2157-2518.S9-004

Page 3 of 4

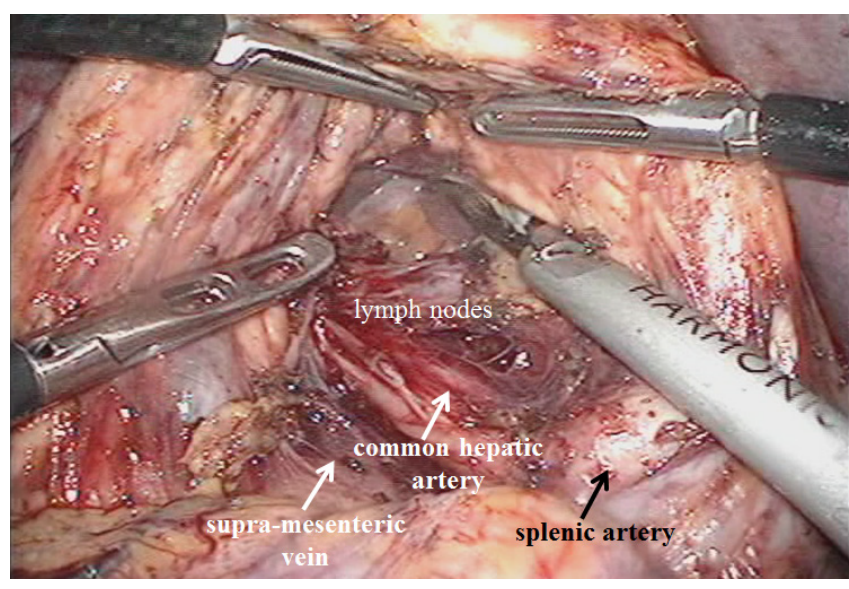

Figure 2: Lymph node dissection. Following the tunneling is performed, the lymph nodes can be dissected from the behind the pancreas.

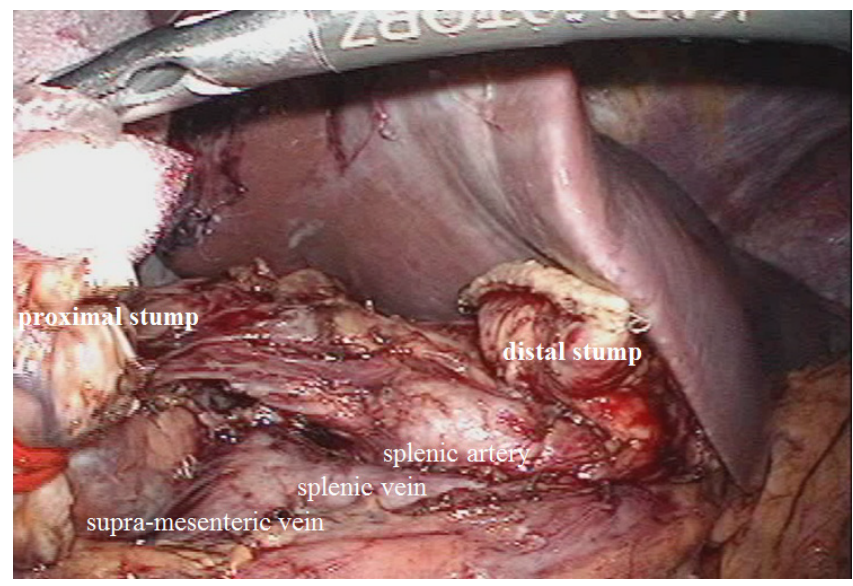

Figure 3: Pancreatic transection in Lap-PD. In order to prevent tumor cell seeding caused by the dispersal of pancreatic fluid after the pancreas transection, we use an endoscopic linear stapler when severing the pancreas and closing the severed pancreatic stump.

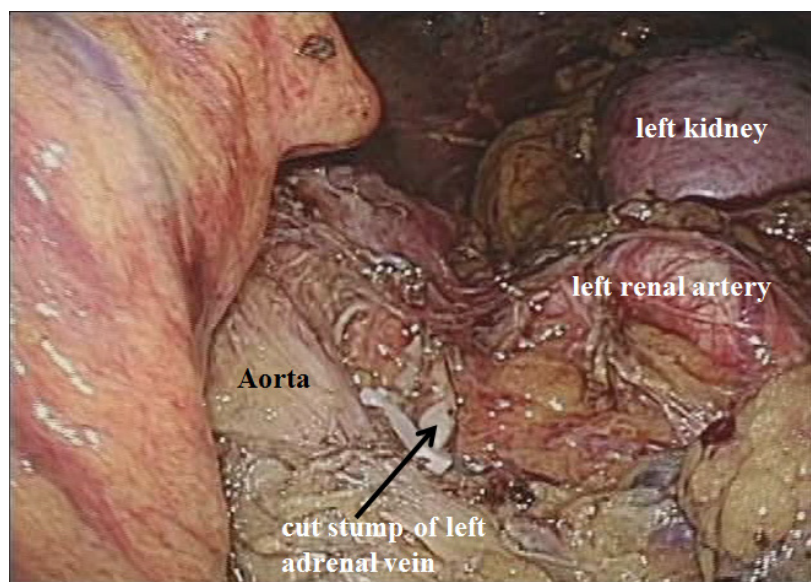

Figure 4: Retroperitoneal tissue dissection. In case with need for retroperitoneal tissue dissection, we can broaden the operative field more than with open distal pancreatectomy (open-DP) where the left costal arch overhangs the operative field.

\begin{tabular}{|l|l|}
\hline Period & Jan. $2004 \sim$ Dec. 2012 \\
\hline Cases & $25(10$ males, 15 females $)$ \\
\hline Mean age (range) & $67.3(29-88)$ y/o \\
\hline Disease stage (TMN classification) & stage $0: 3$ cases \\
\hline & stage I A: 5 cases \\
\hline & stage I B: 2 cases \\
\hline & stage II A: 5 cases \\
\hline & stage II B: 9 cases \\
\hline & stage III : 0 \\
\hline Mean number of retrieved lymph nodes & stage IV : 1 case \\
\hline R0 resection ratio & $22.4 \pm 12.6$ (6-57) \\
\hline Perioperative mortality & $88 \%$ (22/25) \\
\hline Follow-up period (month) & none \\
\hline Survival periods in dead cases & $1-71$ (median 16) \\
\hline & 2.5 months (stage IV) \\
\hline & 15 months (stage I A) \\
\hline & 29 months (stage II B) \\
\hline & 33 months (stage II B) \\
\hline & 24 months (stage II A) \\
\hline & 18 months (stage II B) \\
\hline
\end{tabular}

Table 1: clinical characteristics and outcomes of pancreatic duct adenocarcinoma.

$0 \%$ of patients. While short-term oncologic outcomes may be similar to those achieved via an open approach, the long-term oncologic outcomes remain undetermined. There are reports on long-term outcomes by cancer stage, but the small numbers of patients for each stage means it is difficult to evaluate the results. The median followup noted in those five published reports only ranged between 5 and 36.5 months (mean 20.0 months), so investigations into long-term prognoses are the next step [4-8].

One of the characteristics of PDAC is the high frequency of infiltration into the pancreas nerve plexuses. Therefore, the dissection of pancreas nerve plexuses is just as important as lymph node resection. We think our laparoscopic left mesenteric approach is an adequate and reasonable method that allows laparoscopic dissection of the pancreas nerve plexuses as performed with open approach.

We use ELS when severing the pancreas in Lap-PD and have previously reported our method for closing the resected pancreatic stump [3]. Our method aimed at preventing tumor cells, suspended in the pancreatic juice within the pancreatic duct, from leaking intraperitoneally after the pancreas transection. At present, Lap-PD takes longer than open pancreaticoduodenectomy and laparoscopic approach requires insufflation of the abdominal cavity that results in increased abdominal pressure. We therefore developed this method to address concerns over the increased likelihood of metastasis through peritoneal seeding.

\section{Conclusions}

Our laparoscopic surgery to treat PDAC patients seems to achieve equivalent curability to open approach. We think laparoscopic surgery could be particularly useful in the treatment of PDAC, because this surgery is less invasive and patients may receive sooner adjuvant chemotherapy after the operation.

The long-term outcomes we have achieved by laparoscopic pancreatectomy in PDAC patients are similar to open approach, but we obviously need to continue our research in more patients and carefully continue the follow up, including those patients receiving chemotherapy. 
Citation: Nakamura Y, Matsushita A, Sumiyoshi H, Yamahatsu K, Aimoto T, et al. (2013) Laparoscopic Pancreatectomy for Pancreatic Cancer. J Carcinogene Mutagene S9: 004. doi:10.4172/2157-2518.S9-004

\section{References}

1. Nakamura Y, Uchida E, Aimoto T, Matsumoto S, Yoshida H, et al. (2009) Clinical outcome of laparoscopic distal pancreatectomy. J Hepatobiliary Pancreat Surg 16: 35-41.

2. Nakamura Y, Uchida E, Nomura T, Aimoto T, Matsumoto S, et al. (2009) Laparoscopic pancreatic resection: some benefits of evolving surgical techniques. J Hepatobiliary Pancreat Surg 16: 741-748.

3. Nakamura Y, Matsumoto S, Matsushita A, Yoshioka M, Shimizu T, et al. (2012) Pancreaticojejunostomy with closure of the pancreatic stump by endoscopic linear stapler in laparoscopic pancreaticoduodenectomy: a reliable technique and benefits for pancreatic resection. Asian J Endosc Surg. 5: 191-194.

4. Dulucq JL, Wintringer P, Mahajna A (2006) Laparoscopic pancreaticoduodenectomy for benign and malignant diseases. Surg Endosc 20: $1045-1050$

5. Palanivelu C, Jani K, Senthilnathan P, Parthasarathi R, Rajapandian S, et al. (2007) Laparoscopic pancreaticoduodenectomy: technique and outcomes. J Am Coll Surg 205: 222-230.

6. Pugliese R, Scandroglio I, Sansonna F, Maggioni D, Costanzi A, et al. (2008) Laparoscopic pancreaticoduodenectomy: a retrospective review of 19 cases. Surg Laparosc Endosc Percutan Tech 18: 13-18.

7. Kendrick ML, Cusati D (2010) Total laparoscopic pancreaticoduodenectomy: feasibility and outcome in an early experience. Arch Surg 145: 19-23.
8. Ammori BJ, Ayiomamitis GD (2011) Laparoscopic pancreaticoduodenectomy and distal pancreatectomy: a UK experience and a systematic review of the literature. Surg Endosc 25: 2084-2099.

9. Baker MS, Bentrem DJ, Ujiki MB, Stocker S, Talamonti MS (2009) A prospective single institution comparison of peri-operative outcomes for laparoscopic and open distal pancreatectomy. Surgery 146: 635-643.

10. Jayaraman S, Gonen M, Brennan MF, D'Angelica MI, DeMatteo RP, et al (2010) Laparoscopic distal pancreatectomy: evolution of a technique at a single institution. J Am Coll Surg 211: 503-509.

11. Kooby DA, Hawkins WG, Schmidt CM, Weber SM, Bentrem DJ, et al. (2010) A multicenter analysis of distal pancreatectomy for adenocarcinoma: is laparoscopic resection appropriate? J Am Coll Surg 210: 779-785, 786-7.

12. DiNorcia J, Schrope BA, Lee MK, Reavey PL, Rosen SJ, et al. (2010) Laparoscopic distal pancreatectomy offers shorter hospital stays with fewer complications. J Gastrointest Surg 14: 1804-1812.

13. Mehta SS, Doumane G, Mura T, Nocca D, Fabre JM (2012) Laparoscopic versus open distal pancreatectomy: a single-institution case-control study. Surg Endosc 26: 402-407.

14. Fisher SB, Kooby DA (2013) Laparoscopic pancreatectomy for malignancy. $J$ Surg Oncol 107: 39-50.

This article was originally published in a special issue, Pancreatic Ductal Adenocarcinoma handled by Editor(s). Dr. Toshiyuki Ishiwata, Nippon Medical School, Japan 